\title{
AUTOMATION OF A GEOSCAN ELECTRON MICROPROBE
}

\author{
T. A. Ḧ̈KLI and Kalevi Ripatti
}

\begin{abstract}
HäkLI, T. A. and RipatTI, Kalevi 1974: Automation of a geoscan electron microprobe. Bull. Geol. Soc. Finland 46, 105-108.
\end{abstract}

A Geoscan Mark I electron microprobe has been automated by means of stepping motors and a Nova 1210 computer with $8 \mathrm{~K}$ of memory. The on-line computer controls the movements of the spectrometers and performs the corrections for the absorption and atomic number effects.

T. A. Häkli and Kalevi Ripatti, Outokumpu Co, P.O. Box 27, SF-02101 Tapiola, Finland.

\section{Introduction}

The importance to the exploration and exploitation of mineral resources of the elemental data from rocks and minerals is widely recognised. Consequently, laboratories are faced with ever increasing multitudes of samples at the same time as they are fighting against rising instrumental and labour costs.

An electron microprobe has been in operation as an integral part of research projects at the Geologic Laboratory, of the Outokumpu Co for almost a decade and great number of specimens have been, and thousands more will be analysed by ridged routine. Under these circumstances the feasibility of converting the manually operated Geoscan Mark I electron microprobe into computer controlled automated device was seriously considered. After some preliminary tests the actual plan materialised as a joint venture of the Geological Laboratory, the Physics Research Laboratory and the Engineering Division of the Outokumpu Co.

\section{The instrumentation system}

The system features a Nova 1210 computer with $8 \mathrm{~K}$ of memory and a teletypewriter model ASR 33 for input/output (Fig. 1). The interface boards in the computer rack include a digital input board (16 bite), a digital output board (16 bite), and an interface board for two stepping motors. The rack also houses a power supply for stepping motors and interface boards. The schematic of the system is depicted in Fig. 2.

Two spectrometer servo command units were converted to computer control ones by installing a stepping motor, gear unit and electromagnetic clutch (Fig. 3). The rotation rate of the stepping motors is adjustable. At present, it is about 180 steps per second and a complete revolution requires 200 steps.

Switches have been installed parallel to the start and contact switches for the digital output board. Moreover, there is a switch for starting the counting operation and another one for reading the intensities into the computer. The 


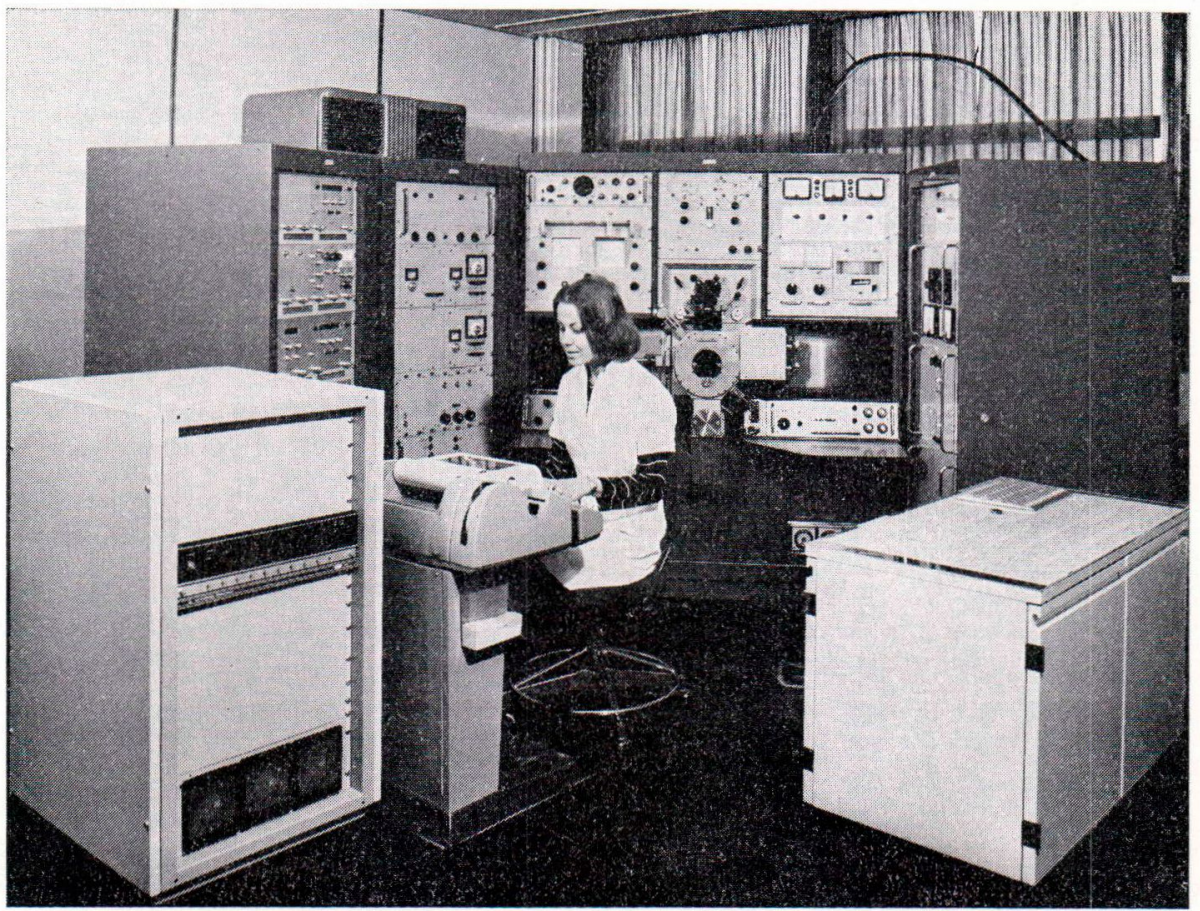

Fig. 1. The automated Geoscan electron microprobe system. The panel of the front left hand side houses the Nova 1210 computer.

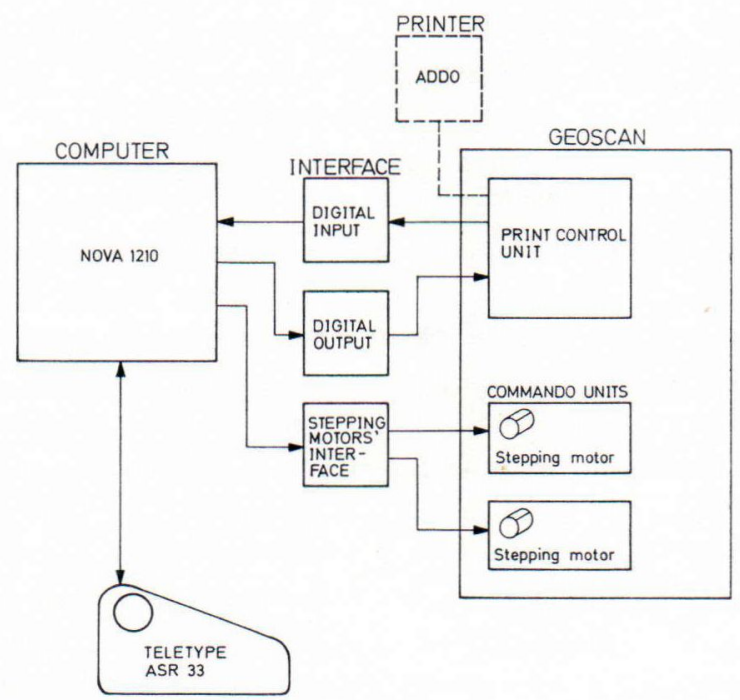

Fig. 2. A block diagram of the automation system. 


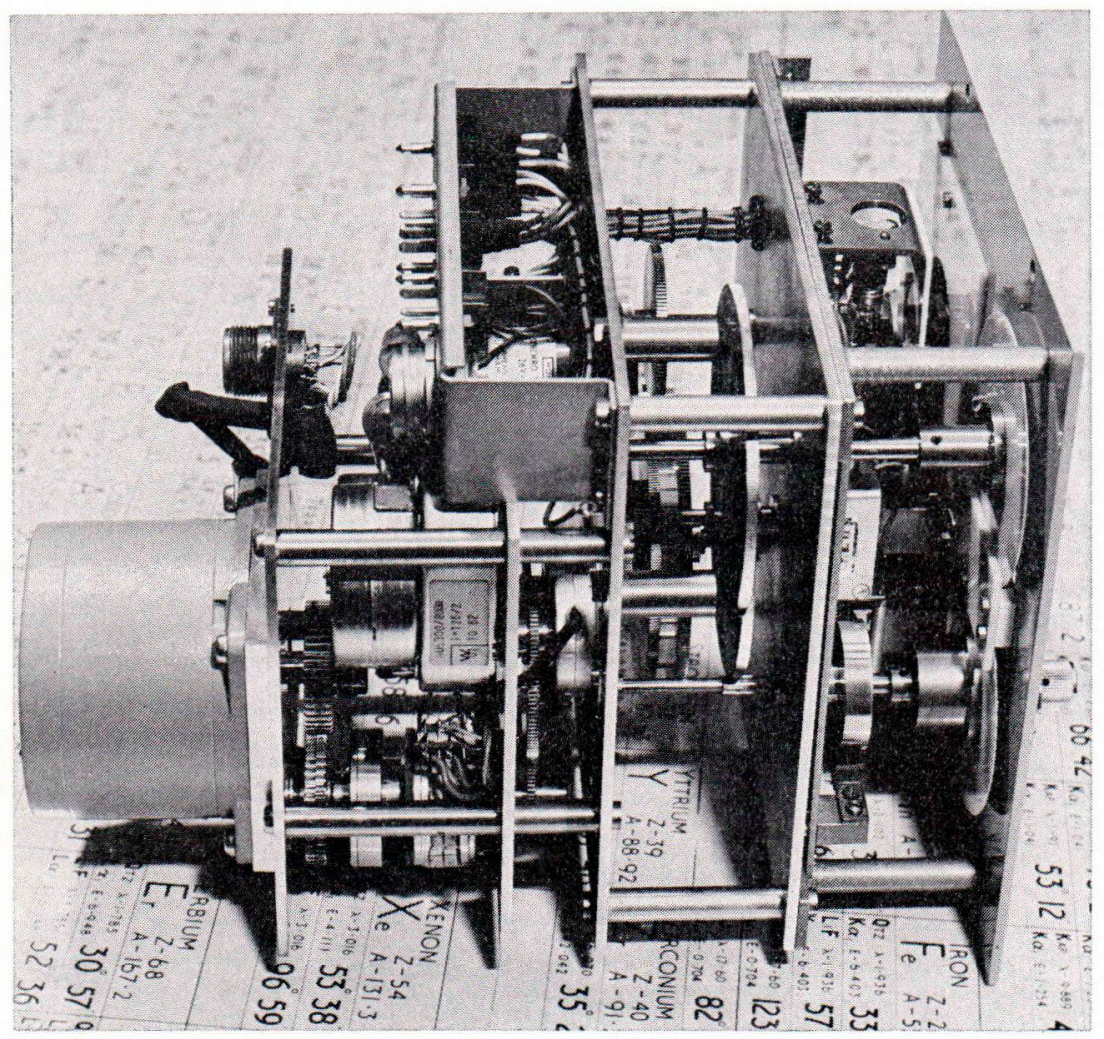

Fig. 3. A command unit with an attached stepping motor.

interpretive system of the Nova 1210 computer is the »Minibasic» or »Su basic with call» developed by the Strömberg Co on the basis of the program N:o 2 »Basic interpreter» of the Users Group of the Data General Corp.

The digital input/output operations as well as the turning of the stepping motors are effectuated with statements written in the Assembler, which can be recalled from the Basic by means of the Call command. The operation program proper is written in the Basic and controls the rotation of the stepping motors, starts the counting procedure, reads the intensities, processes the data, etc. The memory available is shared by three functions: Minibasic c. $3.7 \mathrm{~K}$, interfaces c. $0.6 \mathrm{~K}$ and operation program c. $3.8 \mathrm{~K}$.

\section{Modes of operation}

The equipment can be operated in three ways. The continuous mode is used merely for qualitative analyses, in which case the automatic control is disengaged and the electron probe operated manually. The peak mode, which has found wide application in automated X-ray spectrometers, has turned out to be less suitable for the Geoscan owing to the drift of the peak position. This is langely attributable to the change in temperature in the spectrometer chamber, which slightly alters the geometry of the spectrometers. However, this drawback can be overcome by programming the spectrometers to search for the maximum intensity. But this 
is not very practicable and, hence, most of the runs are done by using the profile mode, which allows a slight shift in the peak position without endangering the analytical accuracy. The goniometers pass stepwise across the peak in this mode of operation. The analyst keys the angle value for each line to be measured as well as the number and size of the steps into Nova 1210 . The intensity values of the first and last two steps are averaged and taken as the background intensity, which is subsequently subtracted from the intensity integral over the peak.

\section{One-line computer programs}

The programs in current use allow specimens to be analysed for a maximum of ten elements, five with each spectrometer. Before and after a series of analyses the intensities from the standards are determined. If the compositions of the standards are close to those of the minerals to be analysed no matrix correction is performed. Otherwise the intensities are corrected for absorption and atomic number effects (Birks 1963; Thomas 1963) by keying the pertinent data of the intensity functions, mass absorption coefficients and other parameters into the Nova 1210 .

After 10 iterations the corrected abundances are printed out. The $8 \mathrm{~K}$ of memory is too small for fluorescence correction. However, the storage capasity can easily be doubled, in which case it is also feasible to apply enhancement correction.

\section{Conclusions}

Automation that emphasises software rather than hardware allows flexibility in the choise of the mode of analysis, and the procedures can readily be altered simply by rewriting the programs. Moreover, analytical system can be made as complicated as necessary to ensure optimal accuracy without having to take into consideration obstacles arising from human factors, which play an important role in manual operations. Finally, the increase in capacity leads to lower costs per analysis and this is by no means the least important achievement of the automation.

Acknowledgement - We thank the Outokumpu Co for the permition to publish this paper.

\section{REFERENCES}

Brrks L. S. (1963) Electron probe microanalysis. Interscience Publishers. 253 b.

Thomas P. M. (1963) Outline of a method for correcting for atomic number effects in electron probe analysis. Br. J. Appl. Phys. 14: 397-398.

Manuscript received, January 16, 1974 